\title{
Considerations on Present Production Science Theory and Didactics: The Evident Lack of a Rational Manufacturing Theory
}

\author{
Bruno G. Rüttimann1, Martin T. Stöckli² \\ ${ }^{1}$ ETH Zürich IWF, Zurich, Switzerland \\ ${ }^{2}$ Inspire AG, Zurich, Switzerland \\ Email: bruno.ruettimann@inspire.ethz.ch, stoeckli@inspire.ethz.ch
}

How to cite this paper: Rüttimann, B. G., \& Stöckli, M. T. (2021). Considerations on Present Production Science Theory and Didactics: The Evident Lack of a Rational Manufacturing Theory. Journal of Service Science and Management, 14, 482-501. https://doi.org/10.4236/jssm.2021.145031

Received: August 28, 2021

Accepted: October 8, 2021

Published: October 11, 2021

Copyright $\odot 2021$ by author(s) and Scientific Research Publishing Inc. This work is licensed under the Creative Commons Attribution International License (CC BY 4.0).

http://creativecommons.org/licenses/by/4.0/

\begin{abstract}
Today's universities' manufacturing courses on production system design and analysis of performance dynamics are heuristics than rational-based, far away from a physics or mathematics theorem constructed science. This is also due to increasing computational power, which allowed the proliferation of simulation-based modeling for complex manufacturing systems to analyze their behavior and optimize their performance. Indeed, manufacturing theory remained stuck to generic practice-based production concepts. The lack of a well-structured scientific production specific theory is increasingly problematic. Engineering students lacking the foundation of a solid theory will base manufacturing systems design rather on believing that the optimization software will solve the scheduling issue instead of comparing alternative modern design principles to conceive high performance manufacturing systems. This attitude is far away from a scientific rational approach to design demand characteristic and product appropriate manufacturing systems by knowing "the theory". A scientifically formulated manufacturing theory should cover two aspects: Firstly, a proper Cartesian-based understanding and law-based modeling of manufacturing systems to describe rationally their behavior; and secondly, a library with a comprehensive set of elementary production systems' design principles. The design principles define the functioning of the manufacturing system and the theory models the resulting dynamics of the manufacturing system. This paper exemplarily shows the increased didactic and professional benefit of such an appropriate, solid, theorem and law based manufacturing science. In addition, it also discusses the impact of presently applied manufacturing simulation in today's context of emerging Industry 4.0 type cyber-physical production systems (CPPS).
\end{abstract}




\section{Keywords}

Production Theory, Lean Manufacturing, Industry 4.0, CPPS, System Dynamics, Simulation, Heuristics, Manufacturing Science

\section{Introduction}

"Hic locus est ubi mors gaudet succurrere vitae" is an often seen inscription at anatomic institutes of European universities. This inscription documents that what today is called "heuristic approach" has already been used in the dawn of scientific thinking coming close to the approach of empiric learning gaining experience by doing. Heuristics starts usually from empirically acquired knowledge in absence of science-founded laws by applying a deductive rational logic to gain insights and drawing correct, or sometimes, false conclusions. It is interesting that heuristic approaches are widely used to the present days. This is not negative, however, if systems become too complex, e.g. such as manufacturing systems usually are heuristic approaches that find their limits. Indeed, the lack of a scientific manufacturing theory stresses the divulgation of common isolated practice-derived manufacturing beliefs forcing, without systematic solid foundations, the explorative simulation approach. In such cases, a cognitive model-based theory would allow to put the discussion on the level of a rational science-based understanding. Today, simulation techniques are applied to model, or to explore, the dynamic behaviour of a manufacturing system. There is an evident twofold reason for that: not only the lack of a manufacturing theory but also the complexity of manufacturing systems. Generally, the modelling of a system might be solid law-based or rather exploration focussed, simulating or emulating the behaviour, finding deterministic solution, or optimizing and decision-oriented. It is not the intention of this paper to provide an anthology of simulation techniques and modelling types, but to show that both topics, theory and simulation, are complementarily linked. Nevertheless, to the contrary of manufacturing "science", some natural exact sciences, such as physics or chemistry, have developed their own governing laws mainly supported by the help of mathematic formalism. Mathematics at its own has developed an own exact and structured logic, based on axioms, theorems, and corollaries to be consistent.

This has not been the case for production sciences where theory and the knowledge transfer are still stuck divulging simple empirics-deduced and heuristic-induced approximations or even false manufacturing "theory" concepts, such as the widespread believe "the whole attention should be directed to the most expensive equipment". This often heard statement is correct from a theoretic economic asset point of view, but not from a manufacturing one. In manufacturing, the attention has to be oriented towards the bottleneck, which directly limits productivity. While in the dawn of modern physics rational laws have been formulated directly derived from simple empiric experience, think only at 
Galilei and Newton, Ampère and $\mathrm{Ohm}$, the definition of manufacturing theory curriculum missed to translate empiric gained knowledge into universally valid "production laws". Therefore, graduated engineering students lack the rationality of a science-based theory not only to design but also to discuss the behavioural dynamics of manufacturing systems, which should distinguish them from old school practitioners. To find the reason for that we have to discover the intrinsic difference between manufacturing and physics or chemistry, which is inherent to the topic itself. Indeed, manufacturing is not a natural science, but is a manmade artificial construct of applied concepts. Therefore, it has not primarily the understanding of the logic transformation process as topic, but how the morpho-physical transformation of the object is technically implemented on the shopfloor. This means that manufacturing, and we say expressly not manufacturing science, is rather implementation and solution-oriented and not understanding-oriented, i.e. practical engineering and not theoretical physics based. The pity is that Lean-based manufacturing solutions often are neglected in academic knowledge transfer in favour of generic simulation-based optimization approaches instead of trying to understand "modern" alternative production systems such as the Toyota Production System (TPS). However, what makes manufacturing so different and difficult to be formalized? How is it defined? If you consult Wikipedia about manufacturing you will be disappointed, a misleading definition which we purposely do not cite here, and shows the existing nebulous delirium about the topic. A clearer and more comprehensive definition of a manufacturing system has been given in (Rüttimann, 2017):

"[Manufacturing is] the optimization of a constraint system within a non-deterministic environment

- with the objective to transform raw materials into products (applied technology)

- complying to customer requirements such as on-time delivery (OTD, respecting the voice of the customer)

- having limited process resources available (restricted capacities)

- applying an appropriate allocation, i.e. scheduling of resources (optimal solution)

- showing different throughput and lead-time characteristics (process performance)

- by following the economic rationale of minimizing waste of inputs and resources (ROI)".

Note that product quality is not even mentioned here, because it is presumed to be engineered and delivered at 'six sigma' level to the customer. The same applies for machine downtime, which should be limited to maintenance and set-up. The above definition shows how complex it is to manage a production system, but also why a theory model is not easy to be developed and why it has not yet been configured until today. Indeed, common laws are usually simple, however, man-made manufacturing systems are complex. Such a configuration 
is difficult to be reconciled. To model the complexity of manufacturing, operations research techniques such as linear programming have been deployed to find appropriate production scheduling covering at least a part of the above reported definition. Already the naming of linear programming is a testimonial of the very first applications of linear optimization techniques to solving manufacturing-related planning problems. The predominant applied batch \& queue (B \& Q) manufacturing in Western industries production forcedly called for the application of queuing theory. From queuing theory some elementary calculations such as Little's law, Kingman's and Kuehn's approximations have been derived. To master the complexity of production planning systems, sophisticated production planning software packages have been developed (called MRP, MRP2, ERP) with increased scope, controlling the advancement of planned scheduled production orders to supply customer orders on time. Furthermore, the manufacturing "theory" is often divulged academically by integrating cognitive heuristics based thinking with empirically gained knowledge and applied simulation. Indeed, the development of a proprietary, specific "physics" of manufacturing has been rarely the topic of research. Nonetheless, the scientific community is conscious about the lack of a systematic manufacturing system design approach (e.g. Francalanza, Borg, \& Constantinescu, 2014), but it seems not bother about the lack of a law-based manufacturing theory as long as there is enough computing power. And exactly this shows the prevailing of practical-focussed implementation approach compared to the understanding-focussed theory approach. However, exactly this theory topic once has already been raised by J. Little in one of his papers 30 years ago (Little, 1992). Since then hardly any relevant initiatives have been undertaken. The reason why this topic has not yet been systematically researched, is not only linked to the complex multi-disciplinarity of the topic itself, but also intrinsic to the increased computational performance, enabling and facilitating easy solution finding by simulation and therefore helping to make optimized implementation decisions.

Nevertheless, we have to pay attention not to be controlled by artificial intelligence (AI) logic-based automation without understanding the governing laws. On the contrary, we have imperatively to understand the basic laws governing the outcome. It is inacceptable that students know how to program and use expensive software of manufacturing systems, but they do not know the basics regarding how different production systems work, e.g. sometimes even believing that Lean is summarily defined by 5S, Kanban, and Kaizen. The intelligent task consists in conceiving performant manufacturing systems knowing upfront the approximate resulting behavior of the selected implementation solution. Indeed, physicians perfectly know the celestial laws, nevertheless it is the computer which calculates the planetary trajectories. Manufacturing simulation, however, is not law-based, but often uses discrete event simulation (DES) to explore the solution space. Manufacturing simulation has to be supportive and is not for compensating the lack of knowledge. It should even less be a surrogate to knowl- 
edge, as it is the case today. It is not only recommended, but necessary that production engineers have understood the functioning of a law-based production theory and the consequence of applied production-related principles. Such a new approach is equal to a paradigm shift in production theory didactics and consequent education of modern engineers conferring them a profound knowledge.

In the following, we will show some excerpts of such a law-based production theory and the most recent production-proprietary findings summarized in new, recently published production theory corollaries. The intention of this paper is to promote a rational science-derived didactics based on theorems of manufacturing theory but also to develop further production related theory initiated with the manufacturing text book (Rüttimann, 2017). The superiority of such a law-based theory compared to the present teaching approach will finally become evident.

\section{Deficiencies and Limits of the Present Scholastic Approach in Manufacturing Theory}

The topic has already been touched by the authors writing an overview paper regarding alternative manufacturing systems, but only at introductory level (Rüttimann \& Stöckli, 2020a). Indeed, often quite some confusion exists about the type of manufacturing systems and along with this, or explicitly due to it, the improper usage of language to name things correctly, an issue that is caused by incomplete knowledge transfer and inadequate didactics favouring simulation over theory. Manufacturing concepts and principles lack scientific rigor and are often explained with imprecise fuzzy concepts. Moreover, they are often named in great sounding empty word shells, leading to misunderstanding and creating confusion from inappropriate language. An example is given by the word "pull" being at the base of Toyota's conceived revolutionary lean JIT manufacturing systems. During our lecturing and consulting experience, we noticed the improper usage by professionals of the word "pull" for designating also a "make-to-order" production, probably derived from the commonly and often used diction "manufacturing on customer-pull". This is not completely false, though, because production starts when the order is given. However, this is not the correct usage of the word and its intrinsic meaning; the correct use is to describe how shopfloor production is triggered, hence the importance to define clear implementation principles. An applied "pull" manufacturing principle triggers production from a downstream operation (manufacturing cell) upstream to the previous cell. "Make-to-order" on the other hand is a production principle (the contrary would be "make-to-stock") designating how to interface external customers. JIT could be described as a shopfloor internal mini "make-to-stock" pull-triggered production with ideally no WIP queuing in between the workstations.

Already in 1983 Hall defined JIT as aiming at a stockless production and zero 
inventories (Hopp \& Spearman, 2000). The counterpart of pull is "push" resulting usually in a scheduled downstream sequence of operations with orders queuing in the process forming a WIP, orders waiting to be processed at the next downstream workstation. Also the widely and superficially used diction "flow on pull”, which refers to the flow implementation regarded as black box process, may induce a wrong and commonly seen interpretation that the flow advancement generally is pull-triggered (white box view). Push and pull are manufacturing principles (Rüttimann, 2017) how order advancement is triggered, and is not directly associated to manufacturing systems often intended as Batch \& Queue (B \& Q) or Single Piece Flow (SPF) based manufacturing. A single piece flow, often called 'flow production' or 'one-piece-flow', which is a transfer principle, can either be push or pull-triggered to advance along the line of workstations to completion (white box consideration). Generally we could state, a SPF is push-triggered whereas entire manufacturing cells are pull-triggered. However, which manufacturing principle is appropriately used depends on various factors not discussed in this session. Due to misunderstandings, it happens that flowshop production is associated with pull (however it has not to be pull implemented) and jobshop production is identified with push (that might be generally correct). However, the "shops" are rather identified by the transfer principle, because jobshop (transferring entire small or medium batches) offer higher flexibility not always implementable with a flowshop needing also a specific layout. A flowshop is rather apt for deterministic environment with large lot sizes implemented with a single piece transfer principle. These examples show exemplarily the mess originating from unclear and vague concept as well as imprecise language and finally from poor didactics. Clearly, such a complex topic combined with the fact that there was little advanced theory available places high demand on the didactics.

A principle based categorization concept trying to clarify this mess is shown in Figure 1. It shows that by crossing the two basic implementation principles, namely manufacturing and transfer principles, four main manufacturing modes are emerging, each one can be further detailed. Please note that they are called manufacturing modes and not manufacturing systems, classifying how production is operated. Also the four manufacturing modes are not unequivocally with regard to the entity "operation" (aggregation of work-content into "boxes of logical content" such as a single workstation or an entire manufacturing cell) and therefore not completely free of potential criticism, hence the importance to talk about principles how a manufacturing system is operationally implemented. Indeed, the upper left case "single piece push" could be named improperly "flow on pull" (if black box viewed) although triggered internally by a push transfer (white box view as shown with FIFO capacity). On the other hand, the lower right case "batch on pull" makes sense when black box viewed (as represented); internally the orders might still be pushed through the different workstations. The topic is complex and this is the evidence that it is important to build clearly 
defined, logically structured knowledge, leading finally to correct understanding and an unambiguous discussion. This is the value of a predicate derived implementation and principle-based structured theory facilitating learning, implementation, and communication of production knowhow.

A further example documenting a misunderstanding of fundamentals is given by the wrong, but often encountered diction that lean JIT is a synchronized manufacturing system. This widely divulged belief is wrong, lean JIT is a highly asynchron system. This shows exemplarily the common widespread misunderstanding of intrinsic functioning of the JIT concept. It is a clear evidence of ignorance regarding the intrinsic knowledge of the TPS functioning and is a direct consequence of European academic non-interest regarding the Japanese TPS not understanding its profound importance in the manufacturing world also for non-automotive industries, in which Lean knowledge usually is limited to some superficial tools notions. Indeed, JIT's asynchrony characteristic derives from Kanban-controlled supermarkets, which separate upstream supply from downstream demand (Figure 2). This resulted to be necessary, because it is difficult to match two logically complete different governed, but interacting systems (upstream

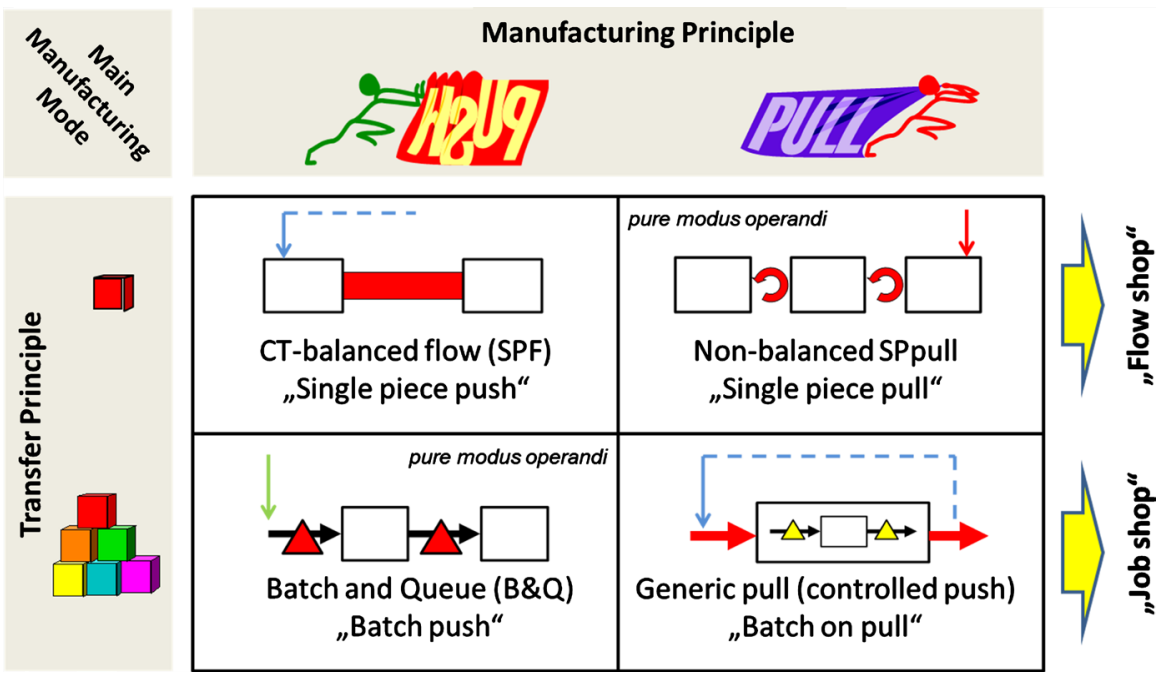

Figure 1. Manufacturing and transfer principles define the main manufacturing mode (Rüttimann, 2017).

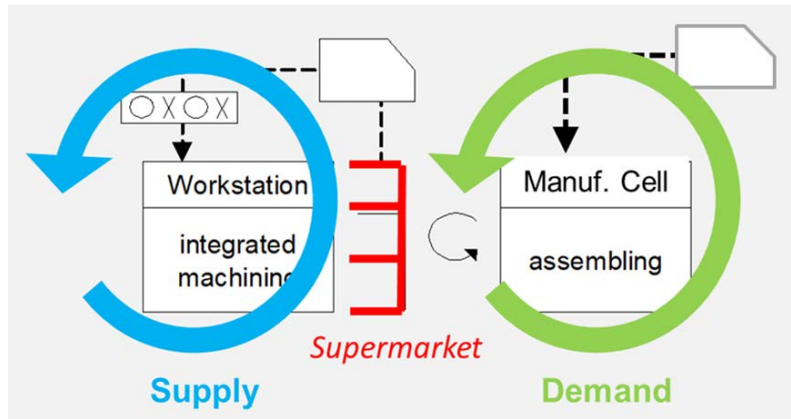

Figure 2. Kanban-controlled supermarket decoupling demand and supply (asynchron system). 
production system with technical supply constraints versus a different downstream withdrawal pace of non-ergodic call-off demand). It represents the quest of Western manufacturing systems, which try to optimize with synchronization and batching as well as simulation and complex planning software for "squaring the circle". We have to pay attention to use the semantics of the word "synchronous" correctly. We have not to mix up the meaning of synchronous with controlled. Lean JIT is Takt Rate (TR) controlled, or TR-governed if you prefer, with the TR being the central governing element of the system. The upstream production is pull-triggered by the downstream product withdrawals out of the supermarket, the supply-loop representing an autonomous system, self-controlled by Kanbans, not needing any operational synchronous production planning nor ERP. This autonomy is an asynchrony characteristic. On the contrary, Petri net modeling is a synchronization technique for general product advancement from the beginning to the end, and original just-in-sequence (JIS) based manufacturing is a visually synchronized systems (where Petri net transactions, or state changes, are imperfectly and JIS perfectly timed). JIS manufacturing is especially applied in final assembly operations to reduce further WIP, because no Kanban managed supermarkets are necessary in that case. Again, the widespread mess of wrong naming originated from the lack of a clear science-based manufacturing theory and is a direct consequence of common fuzzy-approximate, superficially and empiric manufacturing theory and didactics.

We will not screen academic production and manufacturing courses, but give a short overview of presently taught main topics to detect the origin of misunderstanding. Indeed, the teaching scope and focus of production theory usually comprises the following core topics:

1) Description of the different manufacturing processes such as drilling, milling, grinding, rolling, extrusion, casting, etc., each of the technologies accompanied with the relative processing physics applied to different material characteristics.

2) Elements of machine construction and concepts of flexible manufacturing cells or systems (FMS) design, actuators, mechatronics, control and regulation technology, robotics, and programming.

3) Methods of work organization such as MTM (Methods-Time Measurement) and shopfloor layout modeling techniques with increased virtual reality $3 \mathrm{D}$-modeling of physical processes.

4) Operations research theory for optimal capacity planning such as linear programming based on standard linear optimization algorithms or more sophisticated decisional resolutions techniques.

5) Queuing theory for optimal push-based scheduling sequences by applying discrete simulation packages as well as the description of practical applications with MRP2/ERP production planning systems.

What is missing in this enumeration is a dedicated manufacturing proprietary theory block explaining the "physics" of manufacturing going far beyond general 
queuing theory. Indeed, by applying elements of the above "theory mix", the consequent resulting design and construction of a programmable FMS is only a practical case of applied engineering exercise ("applied manufacturing”). Simply said, it is an exercise of machine system design, but not production system design. However, the "physics" of applied implementation principles are not touched. Indeed, such FMS may be operated isolated or in a transfer line (TFL), batch or single-piece transferred, push or pull-triggered, on demand or with centralized or point of use (POU) inventories, with dedicated or common queues, i.e. based on different implementation principles. The behavioural consequence of interconnected workstations (or FMS) versus isolated operated workstations and resulting downstream performance of queued orders, i.e. the comparative performance, is not explicitly known, because a specific manufacturing theory is not existing. The huge difference in performance according to the applied implementation principles is simply not known, because a predicate-derived logic in theory is usually not addressed ("theoretical manufacturing”). It makes a difference which transfer principle is applied. Indeed, specific manufacturing laws, how the entity "order" performs regarding the cardinal importance of process lead time and throughput as well as on-time-delivery performance are often not treated. This means that the laws how the orders advance on the shopfloor, the "factory physics" to use the words of Hopp and Spearman (Hopp \& Spearman, 2000), is not exactly known and often limited to the push-based queuing theory of the trivial Little's law and Kingman's approximation. The reason is simple: the detailed "physics" has not yet been analytically researched and even less formally described, but only experimentally simulated ("simulated manufacturing").

This negligence of an adequate theory in combination to the superficial divulgation of JIT manufacturing systems at many European universities, at least clearly evident in Switzerland, has led consultancy enterprises to fill this vacuum with lean manufacturing training courses. These Lean-"theory" courses have a rather practical connotation and are mainly tools-oriented and do not address sufficiently the theoretic aspect behind modern JIT manufacturing system. Therefore, the value of a rational Cartesian, law-based science ("theoretical manufacturing") is given by the way how students learn. Students have a different learning approach than experienced adult professionals ("heuristic manufacturing"), which can be best described by the "dry sponge vs. wet sponge" allegory. Students need first to build a cognitive structured framework logically interconnected, usually given by theorem and corollaries on which to base their logic decisional scheme (dry sponge approach). Professionals base on their lived experience, and being more critical towards new concepts, they are screening new information before adoption in order to avoid an "overflow of memory" (wet sponge approach). Clearly, both groups need the correct usage of expressions and definitions to profoundly understand the "mechanics" of productions systems. 
Hence the need to evolve today's knowledge transfer from heuristic production aphorisms to a solid comprehensive theory of manufacturing. A paradigm change in manufacturing theory didactics finally is needed: From experimental learning by doing towards implementation by applying theory. We will not enter further into cognitive learning psychology, leaving this topic to the experts, but we think that the core message of the idea has been passed.

\section{Elements of an Insight Gaining State of the Art Manufacturing Theory}

Following a preceding paper (Rüttimann, 2015), which compares the definition of the bottleneck in linear programming with that of lean manufacturing, the very first book tackling such a before mentioned rational manufacturing theory is given by (Rüttimann, 2017). This textbook develops an academically conform format of rational and structured, model-based description of a TPS-derived lean JIT manufacturing system, much different than most of the common lean tools describing books. Exactly such books document that the theoretic dimension of Lean has not yet been understood. However, throughout of the textbook several general manufacturing valid concepts formalized with equations and manufacturing proprietary laws described by theorems, corollaries, and lemmas facilitating a structured learning are developed and applied. This has been necessary, because in lean TPS the topic of queuing theory and production planning is only of negligible importance. Hence, queueing theory is even not necessary. Indeed, Lean is only an alternative way to produce, based on downstream triggered, autonomous SPF manufacturing cells interconnected by Kanban-managed supermarket forming a JIT production system. For instance, the first chapters of this textbook are developing generally valid important production concepts. Very basic statements define and clarify unequivocally the fundamental words of bottleneck, time trap, and constraint, concepts which are at the basis of the "manufacturing physics". These concepts derive from Goldratt's work "Theory of Constraints". Goldratt unfortunately missed to think it to the end and to formalize it. As a consultant, he remained stuck in the practical business-oriented "consultant mode". Furthermore, the dynamics of WIP formation related to time-traps is analytically modeled. Instead of simulating, Rüttimann (2017) formalizes various lead-time calculations for different transfer principles with mathematical equations, as well as defines the necessary and sufficient conditions to supply a customer order on time. Nevertheless, among other clarified concepts and defined principles, finally also a scientific-based definition of "flow" is given that goes far beyond commonly divulged figurative descriptions. This more academic definition of a single piece flow (SPF), by the way directly derived from the divergence operator of vector mathematics, is unmistakable chiseled in the following equation

$$
\text { SPF }:=\left\{\lim _{n \rightarrow 1} \operatorname{Push}\left\{B_{k}(n)\right\}=\lim _{n \rightarrow 1} \operatorname{Pull}\left\{B_{k}(n)\right\} \mid \mathrm{CT}_{i}=\mathrm{CT}_{i+1}\right\}
$$


and described by a theorem and its corollary

\section{Central Limit Theorem of Manufacturing CLTM (or Definition of Per- fect SPF or Manufacturing Principle Identity Theorem)}

In the case of a balanced manufacturing line with equal cycle times $\mathrm{CT}$ at each work station, when the transferred unit tends to one, the two manufacturing principles Push and Pull become indistinguishable, defining a perfect balanced SPF.

\section{Corollary to the Central Limit Theorem of Manufacturing (Corollary of} Improper SPF)

If the transferred quantity tends to one, but the cycle times CT are not equal (unbalanced line), this condition defines an improper SPF (or SP handling); the manufacturing principle push or pull may still be distinguished and depends from the triggering.

With a little imagination, the balanced CT concept of the CLTM shows parallels to the result of the divergence operator in vector fields, when the divergence becomes zero, i.e. the analyzed spatial point is neither a sink nor a source. And exactly this is paradigmatically reflected by the push-pull Manufacturing Principle Identity Theorem. This interesting comparison and, at the same time, astonishing results show exemplarily the value of a rationally exact interpretation of SPF. Indeed, the theorem gives far more insight than present approximate divulgation of a trivial, but figurative description regarding a SPF such as "make-one, move-one", which could also refer to an improper flow (where the divergence in that case would not be zero) and where it is still possible to distinguish an operation as source or sink. The corollary shows the importance of the manufacturing principle, defining the type of triggering for order advancement. The fundamental importance in production of SPF manufacturing compared to B \& Q manufacturing is also reflected by the Theorem of Generalized Lead Time (or Speed Theorem) and its corollaries and lemma, which we will not report here and refer to (Rüttimann, 2017). However, we will report another, directly from the Speed Theorem derived theorem, showing exemplarily the solid structure of the presented manufacturing theory developed in this textbook.

\section{Main Theorem of Production Time (or SPF Dominance Theorem)}

Regardless of the characteristic of a manufacturing system, i.e. with balanced or with unbalanced cycle times CT, the manufacturing lead time MLT is always shorter for a single piece flow SPF transfer principle than for a batch and queue B\&Q transfer principle.

\section{Corollary to the Main Theorem of Production Time (Corollary of Lead Time Limit)}

In the case of a CT balanced line, the SPF principle presents the shortest achievable MLT and therefore also the shortest achievable PLT.

It might be controversial to common beliefs, but the related lemma, the Lemma of SPF Regime, recommends to install also for non-balanced lines a single 
piece transfer principle, because the ER is given by the bottleneck, but the result is that the MLT will be shorter. It becomes evident that a theorem-based learning is the appropriate approach for students to learn manufacturing science in a modern, structured and logic way. This will result in up-to-date and state-of-the-art trained knowledgeable manufacturing engineers. We hope, or suppose with large imagination and goodwill, that experienced manufacturing engineers and production managers, so-called practitioners, who already know the theory not as theorems, but implicitly somehow through many years of experience and applied heuristics. However, the difference lies, and that is very important, not only in the didactics of solid learning, but also in the solid approach of theory-related fact-based competent arguing versus an approximate hear-say-deduced heuristics and experience related superficial chatting.

Apart from this law-based explication of manufacturing theory, manufacturing is made up of a logically and physically implemented "modus operandi". We even intend to go beyond design approaches as described in (Francalanza, Borg, \& Constantinescu, 2014) by first addressing merely the basic elementary principles how a manufacturing systems could be implemented, i.e. how it works (Rüttimann \& Stöckli, 2020a). We have already seen that the production triggering can be classically upstream-push or downstream-pull triggered, principles, which we have named manufacturing principles. If we manufacture specific to customer order or generally to stock, these are called the production principles. The transfer principle defines whether we manufacture batch, single piece or n-piece-wise. All the principles are deliberately selectable by the manufacturing engineer and production manager from a "library" containing a set of proven implementation solutions based on the product and equipment characteristics; they are not subject to theorems. However, the implemented solution will perform according to the manufacturing-specific theorems in terms of ER, WIP-formation, PLT, and OTD. Apart from these main principles, secondary principles are existing, which, as the main principles, can always be selected appropriately and intelligently by the systems engineers, i.e. the designers of the manufacturing system, such as:

- scheduling principle (e.g. FIFO, LIFO, EDD, SPT).

- debottlenecking principle (e.g. spreading, sequentialization, parallelization).

- queuing principle (e.g. common queue, specific queue).

- allocating principle (1-to-1, 1-to-m, k-to-1, k-to-m).

- balancing principles (fixed zone, dynamic zone, circuit, static).

- storage principle (e.g. random, affinity, frequent use).

- location principles (e.g. centralized, decentralized, POU).

- etc.

Such a structured manufacturing system design approach shows how universities might help the industry by forming well-prepared engineers, or more precise, how science-based knowledge didactics supports decision-oriented model application. Mother nature may originate skilled omniscient geniuses, who are capable to design perfect systems not needing to understand performance laws. 
Universities, on the contrary, should generate rational thinking engineers, which are capable to logically conceive perfect systems based on solid knowledge knowing ex-ante the approximate behavioral outcome. That the theory described in (Rüttimann, 2017) is not yet comprehensively developed is shown by an additional enounced implementation principle developed in (Rüttimann \& Stöckli, 2021) dealing with the equipment set-up time of a multi-stage manufacturing cell: the Set-up principle (after-emptying set-up, during-emptying set-up). Lean experts know it, because this is implicitly obvious in lean-managed manufacturing systems, but it is not generally divulged in classic B \& Q manufacturing. Lean has addressed many optimization topics, which could also be applied in traditional manufacturing, but one needs to know the lean TPS. The necessity to explicitly define this principle emerged at the occasion of modeling the backlog (BL) and backlog waiting time (BWT) of queued production orders (Rüttimann \& Stöckli, 2021). The explicit distinction of the applied setup principle is important to formulate the performance equations correctly. This approach reveals that describing alternative implementable technical solutions for manufacturing gives a framework to structured manufacturing systems engineering, which is above of present vague and loose concepts enumeration: from experienced-based implementation to theory-based systematic design. The set of principles form a sort of $\mathrm{n}$-dimensional hyperspace from which one $\mathrm{n}$-tuple constitute the selected variant of each single principle used for implementing the manufacturing system. Lean JIT manufacturing system is only one combination of this n-tuple, the same is also valid for B \& Q, Ford's SPF mass-production, or graph-based CPPS. Apart from the possibility to compare the performance of various manufacturing systems, such a principle-based and structured production engineering approach combined with production specific theorems finally elevates the manufacturing topic from heuristics to a science.

The textbook (Rüttimann, 2017) sets the foundation of a new modern theory, which is based on a rational approach, it is so called "predicate"-derived, i.e. functionally logic, with the most important theorems of production now defined. However, the topic can be considered to be still in a near virgin state. Indeed, based on one of the most important theorems for production theory, which is the Theorem of General Production Requirements (or OTD Theorem) introducing the necessary and sufficient conditions to supply customers on time, additional corollaries have been developed by the authors in (Rüttimann \& Stöckli, 2021) and (Rüttimann \& Stöckli, 2020b). It is incredible, but for decades production managers talk about OTD or OTIF (on time in full), but nobody has ever thought about the necessary and sufficient conditions of manufacturing systems to supply orders on time. Indeed, only in 2017 this OTD theorem has been directly derived from the SPQR axiom (Rüttimann, 2017). The SPQR axiom sets the elementary and non-discussable customers' general requirements of speed, punctuality, and quality, as well as the shareholders' indispensable requirement of economic return for a manufacturing system, forming the minimal necessary basics for a business viable economic production system. The impor- 
tance of the SPQR axiom for manufacturing can be compared to Peano's basic axioms in mathematics or to the two alternative fundamental actions of behavior in economics to resource optimization: "maximizing output with a given input, or minimize input to obtain a required output". The SPQR compliant fundamental OTD theorem states that to be on time

\section{Theorem of General Production Requirements (or OTD Theorem)}

The necessary and sufficient conditions to supply a customer with OTD, i.e. with the right quantity at the right time, is that first the capacity requirement and second the lead time requirement have to be satisfied simultaneously, independent of the applied transfer principle, i.e. SPF or B \& Q. The capacity requirement is given by the Corollary of Weak WIP Stationarity and the lead time requirement necessitates that MLT plus BWT is shorter than EDT.

and can be expressed mathematically according to

$$
\begin{aligned}
& \text { for }: \mathrm{SD}[\mathrm{OR}]>0 \\
& \left\{\begin{array}{l}
\forall i: \inf \left\{\mathrm{ER}_{i}\right\}>E[\mathrm{OR}] \\
Z: \mathrm{BWT}_{Z}+\mathrm{MLT}_{Z} \leq \mathrm{EDT}
\end{array}\right.
\end{aligned}
$$

where OR stands for order rate and $\mathrm{ER}_{i}$ for exit rate of each processing step, MLT for manufacturing lead time of producing the whole batch, EDT the expected delivery time of customers, and BWT the backlog waiting time of the queued orders. The index $Z$ in the equation indicates the order entry point of the process that represents the start of the lead-time for the customer, called customer visible time (CVT). Therefore, the MLT (without index $Z$ ), which starts from the raw materials might differ from the CVT if the entry point $Z$ is not from the beginning of the manufacturing process, but starts from semi-finished components stocked in a supermarket. Typically, the entry point $Z$ could be a customization operation buffered with up-stream standard components of Kanban-managed supermarkets. Although the concepts derive from very evident basic facts of queuing theory, the implicitly made assumptions have never been explicitly enounced in an incontestable manufacturing statement, formalized with a theorem and its equational representation. Equation (1) gives the necessary and sufficient conditions for a manufacturing line to deliver an order on time according to EDT. These explanations shall give a brief introduction, it is not the intention to explain manufacturing theory in this paper. However, they show how a science based manufacturing theory facilitates understanding, and these concepts are only about the basics.

Indeed, in-depth studies (Rüttimann \& Stöckli, 2021; 2020b) regarding the implications derived from non-TR controlled systems, i.e. manufacturing systems governed by a stochastic process of order arrivals, have been leading to additional corollaries to this important OTD theorem. This OTD theorem is probably the most important theorem of manufacturing theory at all. Indeed, quality requirements are lean implicit and can be considered to be met as well as 
$100 \%$ equipment availability is not questioned. The present four corollaries to the OTD theorem are:

- First Corollary to the Theorem of General Production Requirements (Corollary of Post-optimality or Virtual Elasticity).

- Second Corollary to the Theorem of General Production Requirements (Corollary of Strong and Weak OTD Solutions).

- Third Corollary to the General Production Requirements (Corollary of Post-optimal BWT).

- Forth Corollary to the General Production Requirements (Corollary of Ergodic BL Rescheduling).

We will not enter into the matter of these four enounced corollaries and refer to the papers (Rüttimann \& Stöckli, 2021; 2020b), they shall represent a testimony that manufacturing theory is far from being exhaustively defined in (Rüttimann, 2017). A further paper dealing with non-ergodic order arrivals within a non-deterministic product-mix environment is in preparation. With theoretic considerations that paper will set the foundation for modeling CPPS as well as understanding the dynamics and performance of so-called Industry 4.0. Indeed, theory provides knowledge, simulation only provides information.

Although elements from different sciences such as statistics and queueing theory are employed, these corollaries form a part of the framework of manufacturing-specific theory on how orders are logically processed within a manufacturing system. The knowledge does not replace the simulation, but the knowledge allows to understand the real "physics" how orders transit the shopfloor. This systemic knowledge and the appropriate consideration of implementation principles to be selected, combined with the knowledge of the Theorem of WIP and its corollaries, allows knowing "a priori" the approximate dynamics of a manufacturing process regarding throughput, lead-times, and hotspots of potentially arising dynamic time traps. The value of theory-based engineering is uncontestable.

\section{The Value of Appropriate Simulation}

In the foregoing section we have discovered the necessity and benefit for manufacturing engineers of a rational production science. The associated manufacturing theory has the following twofold characteristics:

- production-specific performance laws: these laws explain the throughput and lead time of raw material and semi-manufactured components transiting the shopfloor in order to be manufactured and assembled into finished products (governing "physics" model).

- production-related working principles: these principles offer alternative solutions how a production system can be implemented and how it works. The selection of principles to apply lies in the discretion of the decision maker (applied implementation model). The resulting performance will vary according to the selected principles. 
Please note that ongoing digitalization and resulting CPPSs do not influence the classic implementation principles, and even less the resulting performance. However, also the performance of CPPS will be subject to the basic manufacturing laws defined in (Rüttimann, 2017) and papers (Rüttimann \& Stöckli, 2021; 2020b). Indeed, digitalization will only change how we manage a production system. We have to be aware, if the manufacturing system is poorly conceived, digitalization will only be able to optimize this bad design, hence the indispensable call for theoretic knowledge. It is the configuration of the various implementation principles, which defines the resulting performance in terms of throughput speed according to the manufacturing laws, whether cyber-physically digitalized or not. Furthermore, we have to be conscious that in the case of ERP/APS digital twin (DT) based systems, operational simulation addresses only the topic of scheduling principles and identification of time traps within a defined system of the applied implementation principles. It delivers the order scheduling solution or visualizes the process dynamics for the implemented system. This of course is independent of whether the system was intelligently conceived or not. Prospective production engineers, however, need to master also the theoretical design of modern manufacturing systems using alternative manufacturing principles. This goes beyond the concept of FMS and shopfloor automation, i.e. it is necessary to know the elementary functioning of alternative production systems such as lean JIT systems using FMS or automated processes. By the way, lean JIT is the most performant manufacturing system for a deterministic production mix, but it is not yet widely applied. It is not acceptable that students of production and manufacturing sciences leave university not knowing how to implement such a lean JIT system as it is obviously the case today. If ever, students leave universities only with a tools-based idea of lean, ignoring the well-conceived underlying theory dimension of a JIT system. Also mixed systems (combination of lean JIT and traditional push) may exist. But how can a mixed system be possibly conceived if the design of different sub-systems are not mastered or not even well known. In addition, manufacturing engineers should be aware of the different intrinsic law-based dynamics depending on the selected implementation solutions, in order to increase performance (Rüttimann \& Stöckli, 2020a). This avoids to be blind "prisoners" of acquainted 2D and 3D-simulations. The new theory allows to asses upfront the expected behavior and performance.

A further important topic is the order characteristics. Indeed, a manufacturing system is also designed in function of the order entry characteristics. Knowing the theory allows for controlling in advance the order intake appropriately to match the optimal existing capability of the manufacturing systems. With that the possible order intake can be simulated already under conditions that are compliant to the manufacturing system capability. Indeed, a constrained system with unfavorable conditions eventually cannot be resolved mathematically by optimization simulation. This means that the theory has to dominate the simulation approach. The advantage of a structured manufacturing theory is uncontestable. 
Nevertheless, manufacturing systems are complex dynamically modeled decisional systems showing therefore potentially feedback loops. The representation of dynamic systems with algebraic equations is not always possible, needing infinitesimal modeling. For such characterized systems, hydraulics derived software have been employed, simulating the behavior of dynamic systems, modeling arising eventual instability due to delays in the loops. Today, discrete event simulation (DES) methods for modeling the manufacturing shopfloor are often applied, such as the Tecnomatix plant simulation (Siderska, 2016). To operate such simulation packages in order to analyze the queuing behavior and production advancement of customer orders on the shopfloor, simplified said, potentially one does not need to know the "physics" of throughput, but has only to know the "syntax" of software programming how to map and describe the manufacturing system in a compiler-compliant programming language. The software package will simulate the dynamics without the engineers to have fully understood the functional behavior of the underlying manufacturing system. This lack of theory results in an impoverishment of production student's learning and a patronalization by the software solutions. Moreover it limits the competencies of an engineer already during his curriculum studiorum and consequently later in his professional career. Remember the critics made in section 1 for such an applied approach (simulation vs. theory), we, however, explicitly do not condemn this current simulation. To the contrary, we encourage the use of such software, because of their un-estimated help to implement optimized production scheduling for complex systems for reducing lead-time and guaranteeing OTD. Such complex systems need to be a-priori modeled and live simulated with the help of a software.

Today we are used to work with digital twins representing a specular mapping of the production system on the computer, where alternative scheduling principles may be simulated or brute-force exhaustive alternatives are computed. However, we should talk about a sub-optimal near-exhaustive simulation, because the problem is often of NP-complexity even at the theoretic limit with $\mathcal{\sigma}\left(\sum_{m} m !\right)$ factorial growing permutations of the power set $\wp(m)$ of the alternative addressable machines $\mathrm{m}$. Publication of papers on digital twin-based simulations are proliferating, e.g. (Flores-Garcia, Kim, Yang, Wiktorsson, \& Noh, 2020) and (Barni, Pietraroia, Züst, West, \& Stoll, 2020). However, the contents of these papers are often not adding substantial value, because they are mainly documenting the implementation of a nice practical example of applied production scheduling optimization showing the advantage of the digital twin simulation based on APS (advanced planning and scheduling) compared to classical ERP systems. Nevertheless, these digital twins are a first step with huge potential in SMEs. They will bridge the long way towards the diffuse application of the forthcoming AI-based cyber-physical production systems.

The difference of such Industry 4.0-type manufacturing systems compared to today's "3.0-type", will be the autonomous built-in intelligence of workstations, automated guided vehicles (AGV), and products, applying AI-based learning 
algorithms, communicating via IOT. According to the German i4.0 action group the final aim of CPPS is to have a decentralized self-controlled neural network with direct interaction between the "intelligent" RFID objects even without central coordination unit. Such decentralized intelligent devices (workstations, AGV, products), which, due to the IOT, are also intended to integrate in-bound and out-bound logistics of arriving lorries as well as production planning systems of suppliers and customers. According to the i4.0 action group such systems should even allow customized batch size one, stating literally "to put a Porsche seat into a VW" (Umsetzungsempfehlungen für das Zukunftsprojekt Industrie 4.0, Abschlussbericht des Arbeitskreises Industrie 4.0, 2013). If such omnipotent self-directed systems are economically realistic or subject to "dreams of engineer", we do not discuss here, the well disposed reader already knows the opinion of the authors (Rüttimann \& Stöckli, 2016). However, instead of merely pursuing paraphrased vague CPPS ideas of the i4.0 action group, responsible engineers and industry managers should primarily enforce the integration of DT mapped central production control into present ERP systems. Such production-control integrated DT can propose and implement for the decision maker (production manager) a highly valuable optimal scheduling for OTD, e.g. (Barni, Pietraroia, Züst, West, \& Stoll, 2020). Advanced application may not only lie in twinning the shopfloor status for simulation, but finally oriented for interacting dynamically and directly (autonomously and automatic actuated) or better indirectly (still human controlled activation) with the physical entities, such as workstations, AGV, and products. Indeed, remember Elon Musk's statement at the occasion of robotics generated failures at the Fremont Tesla factory missing the output target: "I had underestimated the value of human workers". The goal must be to advance from CAD/CAM and CIM/FMS to an optimized PPS and computer integrated shopfloor (CIS). This intermediate step of evolution, let us call it "Industry 3.5", might not only be more realistic, but also more desirable than high-flying, today still unrealistic, fully decentralized governed, and expensive AI-controlled CPPS, which are not yet finally conceived and far from being tested, simply because they are not yet existing in their full form. Presently, the attention should rather be focused on the divulgation of APS-like systems. By the way, the idea of the $4^{\text {th }}$ Industrial Revolution goes beyond wearing intelligent goggles displaying virtual images of the shopfloor reality in order to understand the machine status or assist work by visual standard operating procedures. More interesting would be to know up-front the theoretical consequences of the performance of such expensive 4.0 CPPS without complex simulation. Mastering the theory to design performant systems is therefore even more essential. Indeed, "the goggles" will not increase the theoretical performance of the system, but to know the theoretical performance of alternative systems up-front strongly helps to increase ER and reduce PLT, and certainly will limit costs. Furthermore, we have to be conscious that decentralized controlled sub-systems, such as replenishment of warehouse and auto- 
nomous verification of in-bound OTD, may make sense, however for general shopfloor production planning maybe not. Indeed, a centrally optimized and controlled planning system can be Pareto-efficient, a decentralized neuronal controlled system can only be Pareto inferior "Bellman-optimal". This means that due to the underlying theory, the possible solutions simply cannot be as good as the centrally optimized system. It might be, however, that a partly decentralized system produces the best local optimum, like democracy on several levels regarding delegation of authority in well-organized countries.

\section{Conclusion}

As the title of this paper reveals, this is not a scientific paper, but an essay of a dialectic discourse on an improved knowledge transfer in manufacturing theory divulgation to improve the training of manufacturing engineers. We have not only to integrate a heuristics-approach with science-based theorems; we have finally to switch to an advanced comprehensive Cartesian-based rational manufacturing theory. It cannot be the goal to pass, simply stated from the obvious Little's law directly to applied sophisticated simulation and afterwards to decentralized AI-controlled objects without having understood the "physics" of manufacturing. We have not to become slaves of computers and artificial intelligence, no, we have to dominate technology and for that, we have to gain profound knowledge, which leads to the conscious application of explicit design principles. For that, however, we have to know the resulting dynamics of the combined principles, not simply as often rashly cited "per aspera ad astra", but more intelligently "per cognitionis ad astra". The topic of manufacturing theory is complex and by far not exhaustively explored and above all not yet mathematically formalized, however, as demonstrated, absolutely feasible to be modeled with manufacturing-specific laws. Not only will students benefit from a structured theory-based learning approach, but the industry will benefit as well from a structured design of manufacturing systems, production systems which comply with the characteristics of specific product demands. A lot of research opportunities regarding the topic of "theoretical manufacturing" still lie ahead and should be tackled. Instead of "experimental manufacturing" that led to "simulated manufacturing" of costly CPPS, knowledge transfer and didactics of advanced manufacturing should be extended by rationally "theorem-based manufacturing". This will result in a scientific education and finally to well-prepared engineers. However, this is an academic challenge.

\section{Conflicts of Interest}

The authors declare no conflicts of interest regarding the publication of this paper.

\section{References}

Barni, A., Pietraroia, D., Züst, S., West, S., \& Stoll, A. (2020). Digital Twin Based Optimi- 
zation of a Manufacturing Execution System to Handle High Degrees of Customer Specifications. Journal of Manufacturing and Materials Processing, 4, 109. https://doi.org/10.3390/jmmp4040109

Flores-Garcia, E., Kim, G., Yang, J., Wiktorsson, M., \& Noh, S. (2020). Analyzing, the Characteristics of Digital Twin and Discrete Event Simulation in Cyber Physical Systems. IFIP International Conference on Advances in Production Management Systems, Novi Sad, 30 August-3 September 2020, 238-244.

https://doi.org/10.1007/978-3-030-57997-5 28

Francalanza, E., Borg, J., \& Constantinescu, C. (2014). Deriving a Systematic Approach to Changeable Manufacturing System Design. Procedia CIRP, 17, 166-171. https://doi.org/10.1016/j.procir.2014.01.111

Hopp, W., \& Spearman, M. (2000). Factory Physics (Int. ed.). McGraw-Hill.

Little, J. (1992). Tautologies, Models and Theories: Can We Find "Laws" of Manufacturing? IIE Transactions, 24, 7-13. https://doi.org/10.1080/07408179208964220

Rüttimann, B. G. (2015). Discourse about Linear Programming and Lean Manufacturing: Two Different Approaches with a Similar Converging Rational. Journal of Service Science and Management, 8, 85-91. https://doi.org/10.4236/jssm.2015.81010

Rüttimann, B. G. (2017). Lean Compendium-Introduction to Modern Manufacturing Theory. Springer. https://doi.org/10.1007/978-3-319-58601-4

Rüttimann, B. G., \& Stöckli, M. T. (2016). Lean and Industry 4.0-Twins, Partners, or Contenders? A Due Clarification Regarding the Supposed Clash of Two Production Systems. Journal of Service Science and Management, 9, 485-500. https://doi.org/10.4236/jssm.2016.96051

Rüttimann, B. G., \& Stöckli, M. T. (2020a). From Batch \& Queue to Industry 4.0-Type Manufacturing Systems: A Taxonomy of Alternative Production Models. Journal of Service Science and Management, 13, 299-316. https://doi.org/10.4236/jssm.2020.132019

Rüttimann, B. G., \& Stöckli, M. T. (2020b). Exploiting Virtual Elasticity of Production Systems to Respecting OTD Part 1: Post-Optimality Conditions for Ergodic Order Arrivals in Fixed Capacity Regimes. American Journal of Operations Research, 10, 321-342. https://doi.org/10.4236/ajor.2020.106018

Rüttimann, B. G., \& Stöckli, M. T. (2021). Exploiting Virtual Elasticity of Manufacturing Systems to Respect OTD Part 2: Post-Optimality Conditions for the Cases of Ergodic and Non-Ergodic Order Rate with Deterministic Product-Mix. American Journal of Operations Research, 11, 141-165. https://doi.org/10.4236/ajor.2021.113009

Siderska (2016). Application of Tecnomatix Plant Simulation for Modeling Production and Logistics Processes. Business Management and Education, 14, 64-73.

Umsetzungsempfehlungen für das Zukunftsprojekt Industrie 4.0-Abschlussbericht des Arbeitskreises Industrie 4.0, April 2013.

https://www.plattform-i40.de/IP/Navigation/DE/Home/home.html 